\title{
First Results from ATLAS on QCD, Quarkonia, and Heavy-Flavour Physics in proton-proton collisions at $\sqrt{ } \mathbf{s}=7 \mathrm{TeV}$.
}

\author{
Julie Kirk \\ on behalf of the ATLAS Collaboration \\ Rutherford Appleton Laboratory \\ Chilton, Didcot, Oxfordshire. OX11 0QX. United Kingdom \\ E-mail: julie.kirkastfC.ac.uk
}

The ATLAS experiment is designed primarily to address the key issue of electroweak symmetry breaking through searching for the Higgs bosons and other Physics beyond the Standard Model. With the first data it is important to measure expected Standard Model physics processes which will form the background to new physics searches. It is necessary to understand QCD processes in this new energy regime and measurements of charged particle production in minimum bias events and jet production are presented here. First results on quarkonia and heavy flavour physics are also highlighted. 


\section{Introduction}

The ATLAS detector [1] is a large multi-purpose detector operating at the CERN Large Hadron Collider (LHC). In November 2009 the first LHC proton-proton collisions were recorded at a centre of mass energy $(\sqrt{ } \mathrm{s})$ of $900 \mathrm{GeV}$. During 2010 the LHC has operated at $\sqrt{\mathrm{s}}_{\mathrm{s}}=7 \mathrm{TeV}$ and up to $21 \mathrm{st}$ July 2010 ATLAS had recorded $340 \mathrm{nb}^{-1}$ of data with a data-taking efficiency of about $95 \%$. Many detector and trigger performance results have already been produced and are reported elsewhere in these proceedings. Here highlights of the physics results on charged particle production, jet production, quarkonia and top quark events are presented.

\section{Charged Particle Production}

Measurements of charged particle production can be used to constrain phenomenological models of soft hadronic interactions. Charge particle distributions have been measured in ATLAS for events with at least 1 primary charged particle ${ }^{1}$ with transverse momentum $\mathrm{p}_{\mathrm{T}}>500$ $\mathrm{MeV}$ and in the pseudo-rapidity range $|\eta|<2.5$ at $\sqrt{s}_{\mathrm{s}}=900 \mathrm{GeV}$ [2] and $\sqrt{s}_{\mathrm{s}}=7 \mathrm{TeV}$ [3]. The distributions of tracks reconstructed in the ATLAS inner detector were corrected to obtain particle level distributions. Using the charged particle distributions in a diffractive limited phase space $\left(n_{\mathrm{ch}}>5\right)$ a new MC tune of PYTHIA (AMBT1) was generated [4] which better models the charged particle distributions at high $\mathrm{p}_{\mathrm{T}}$ and high multiplicity.

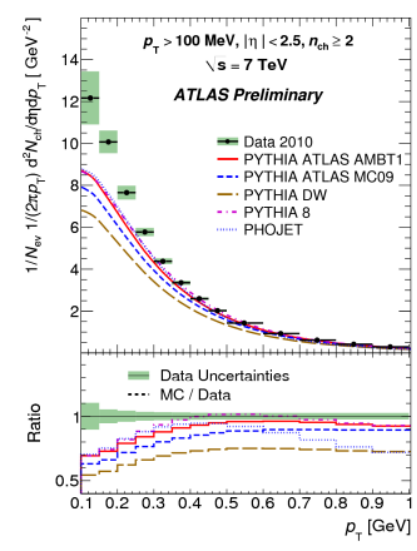

(a)

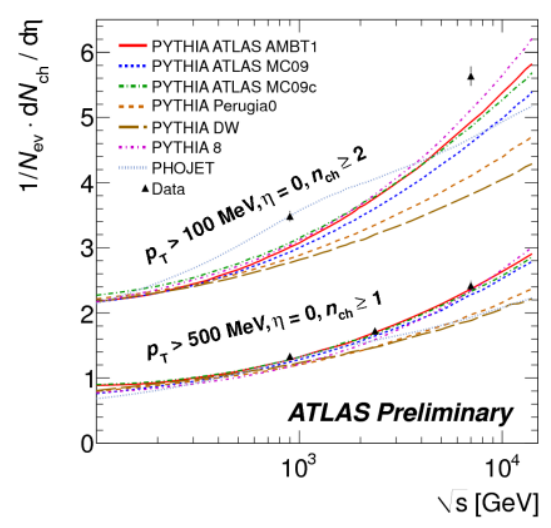

(b)

Figure 1.(a) Charged particle multiplicity as a function of transverse momentum ( $p T$ ) for $\sqrt{ }_{s}=7 T e V$.The data are shown as points with the shaded band showing the statistical and systematic uncertainties added in quadrature. Different MonteCarlo models are shown by the coloured lines.(b)The average charged particle multiplicity per unit rapidity for $\eta=0$ as a function of centre of mass energy.

The analysis has now been extended to include lower momentum tracks $\left(\mathrm{p}_{\mathrm{T}}>100 \mathrm{MeV}\right)$ [5]. For the new analysis, the charged track multiplicity as a function of $p_{\mathrm{T}}$ for $\sqrt{\mathrm{s}}_{\mathrm{s}}=7 \mathrm{TeV}$ is shown in figure 1a) using $190 \mu \mathrm{b}^{-1}$ of data and is compared to different Monte Carlo models. Above $500 \mathrm{MeV}$ the AMBT1 PYTHIA tune agrees well with the data but it starts to deviate in the low pT region. In figure $1 \mathrm{~b}$ ) the charged particle multiplicity per unit of pseudorapidity at

\footnotetext{
${ }^{1}$ Primary charged particles are defined as charged particles with a mean lifetime $\tau>0.3 \times 10^{-10} \mathrm{~s}$, directly produced in $p p$ collisions or from subsequent decays of particles with a shorter lifetime.
} 
$\eta=0$ is shown as a function of centre of mass energy for the analyses using $\mathrm{p}_{\mathrm{T}}>100 \mathrm{MeV}$ and $\mathrm{p}_{\mathrm{T}}>500 \mathrm{MeV}$. The AMBT1 Monte Carlo describes the data less well when the lower momentum tracks are included, some of this difference can be attributed to the larger, and less well understood, diffractive component in this sample.

\section{Jet Production}

The inclusive jet and di-jet cross-sections have been measured [6] using $17 \mathrm{nb}^{-1}$ of data. Jets are reconstructed from calorimeter clusters using the anti- $k_{t}$ algorithm, which is infrared and collinear safe, with a resolution parameter $\mathrm{R}$ of 0.4 or 0.6 . The data are compared to NLO perturbative QCD calculations corrected for non-perturbative effects. Figure 2a) shows the single jet cross section as a function of jet $\mathrm{p}_{\mathrm{T}}$ with $\mathrm{R}=0.4$ and figure $2 \mathrm{~b}$ ) shows the di-jet cross section as a function of di-jet mass with $\mathrm{R}=0.6$. In both cases the data are shown for different rapidity $(|y|)$ bins. The data are well described by the theory. For the data, the dominant systematic uncertainty is due to the jet energy scale, which is below $9 \%$ over the entire range of $\mathrm{p}_{\mathrm{T}}$ and $\mathrm{y}$ and below $7 \%$ for central jets with $\mathrm{pT}>60 \mathrm{GeV}$.

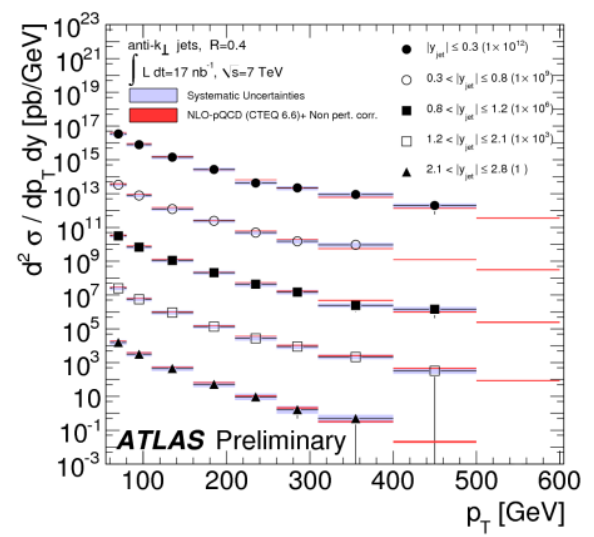

(a)

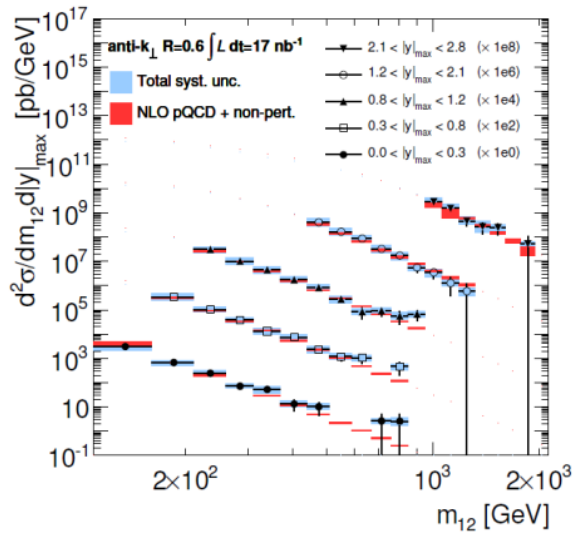

(b)

Figure 2. a) Inclusive jet differential cross section as a function of jet $p_{T}$ in different regions of rapidity $(|y|)$ for jets identified using the anti-kT algorithm with $R=0.4$.b) Dijet differential cross section as a function of di-jet mass, binned in the maximum rapidity of the 2 leading jets The errors bars indicate the statistical uncertainty on the measurement and the grey shaded band indicates the quadratic sum of the systematic uncertainties. An additional $11 \%$ uncertainty due to the luminosity measurement is not shown. The theory uncertainty is shown in red.

A complimentary jet analysis has been performed using charged particle jets [7] where jets are reconstructed using charged particle tracks rather than the calorimeter clusters. This measurement has independent systematic uncertainties and can study lower $\mathrm{p}_{\mathrm{T}}$ jets. Studies of the fragmentation distribution show that it is well described by the AMBT1 Pythia tune for jets above about $10 \mathrm{GeV}$ but below this no Monte Carlo tunes are able to describe the data well.

\section{Quarkonia and charm meson production}

Measuring the quarkonia decays $\mathrm{J} / \psi \rightarrow \mu^{+} \mu^{-}$and $\Upsilon \rightarrow \mu^{+} \mu^{-}$in ATLAS is an important step for understanding the detector and trigger performance as well as for first B-physics measurements. Figure 3 shows the invariant mass distribution for all oppositely charged muons 
in two different mass ranges using $290 \mathrm{nb}^{-1}$ of data. The $\mathrm{J} / \psi$ and $\Upsilon$ peaks are clearly visible as well as the smaller $\psi$ 'peak.
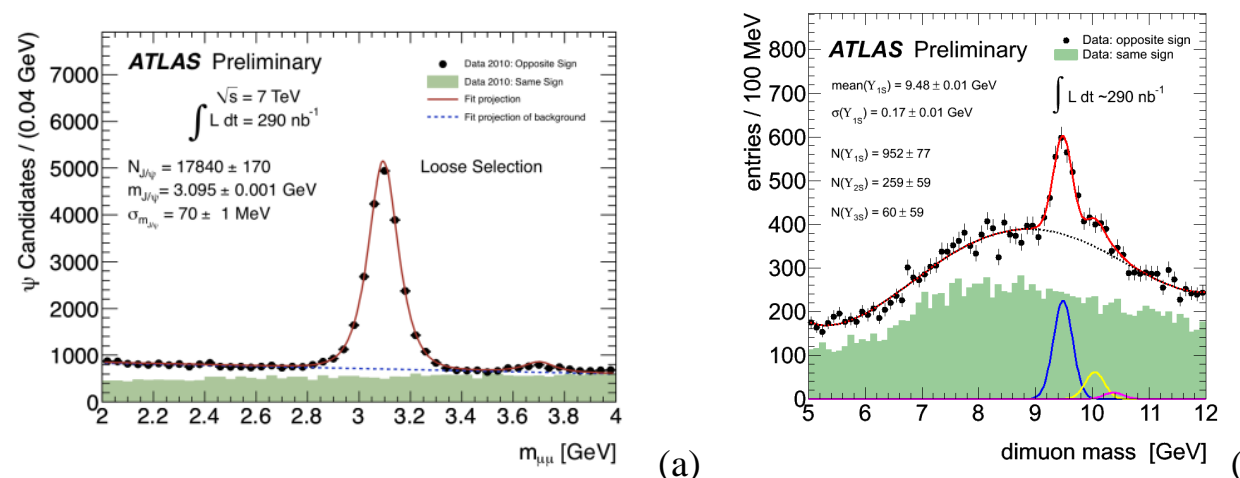

(a)

(b)

Figure 3. Invariant mass of opposite sign di-muon pairs (points) in the range a) 2-4 GeV and b) 5-12 $\mathrm{GeV}$. Same sign muon pairs (green histogram) are superimposedl. The solid line shows a fit to the signal and background, the dashed line shows the background fit.

Two measurements based on the $\mathrm{J} / \psi$ are presented here [8]. The $\mathrm{J} / \psi$ can be produced in non-prompt events via the decay of a long lived particle such as a B-hadron or in prompt decays from short lived sources such as QCD-related subprocesses. The ratio of the number of nonprompt $J / \psi$ to the number of prompt $J / \psi$ is measured in 5 bins of transverse moemntum $\left(\mathrm{p}_{\mathrm{T}}\right)$. In each $\mathrm{p}_{\mathrm{T}}$ bin an unbinned maximum likelihood fit to the pseudo-proper time distribution ${ }^{2}$ is used to extract the ratio from the data. Figure 4a) shows the measured ratio as a function of $J / \psi p_{T}$ showing good agreement with the prediction from the PYTHIA Monte Carlo.

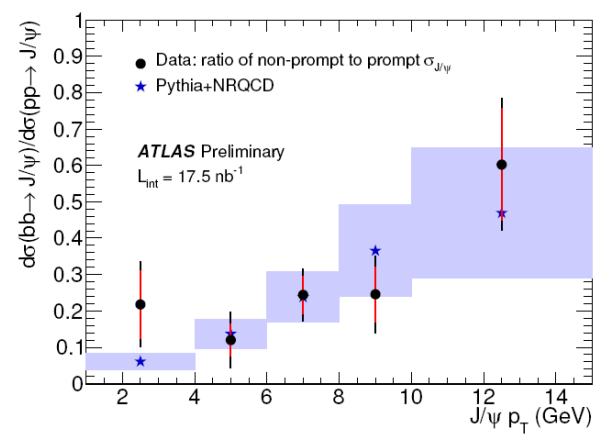

(a)

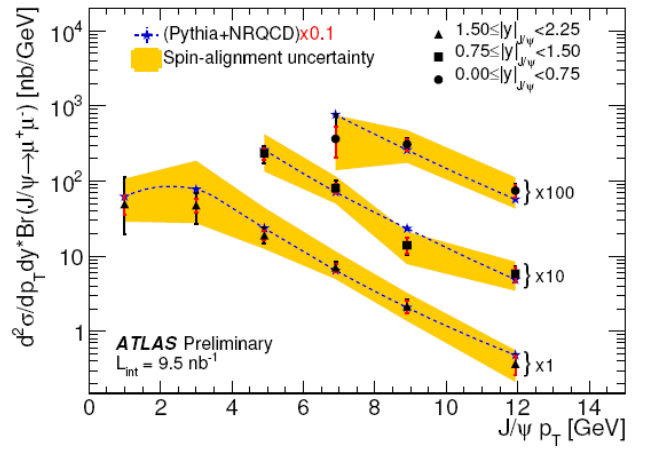

(b)

Figure 4: a) Ratio of non-prompt to prompt $J / \psi$ production cross-sections as a function of the $J / \psi$ transverse momentum. Overlaid is a band representing the prediction from PYTHIA Monte Carlo using various spin-alignment scenarios. b) Corrected inclusive J/ $\psi$ cross-sections as a function of $J / \psi$ transverse momentum and rapidity.

The differential cross-section for $\mathrm{J} / \psi \rightarrow \mu^{+} \mu^{-}$is measured in bins of transverse momentum and rapidity. The events are weighted to account for detector acceptance, reconstruction efficiency and trigger efficiency. An unbinned maximum likelihood fit is used to extract the mass and number of signal candidates in each bin. Figure $4 \mathrm{~b}$ ) shows the results of the crosssection measurement compared to predictions from PYTHIA. The distributions for the different rapidity bins have been multiplied by a scale factor (as indicated on the plot) in order to visually

\footnotetext{
${ }^{2}$ Pseudoproper time $\tau=\mathrm{L}_{\mathrm{xy}} \mathrm{m}(\mathrm{J} / \psi) / \mathrm{p}_{\mathrm{T}}(\mathrm{J} / \psi)$ where Lxy is the xy displacement of the candidate from the primary vertex, $\mathrm{m}(\mathrm{J} / \psi)$ is the mass of the $\mathrm{J} / \psi$ candidate and $\mathrm{pT}(\mathrm{J} / \psi)$ is the transverse momentum of the $\mathrm{J} / \psi$ candidate.
} 
separate the three sets of data. The shape of the distributions are in agreement although the overall normalisation in the Monte Carlo is a factor 10 higher.

The charm mesons $\mathrm{D}^{* \pm}, \mathrm{D}^{ \pm}$and $\mathrm{D}_{\mathrm{s}}^{+}$have been reconstructed using tracks measured in the ATLAS inner tracking detector [9]. Clean signals are observed in all channels and the fit masses are in good agreement with the PDG values. As an example, figure 5 shows the K $\pi \pi$ mass distribution for the $\mathrm{D}^{ \pm}$candidates.

\section{Top quark pair production}

A search for candidate events consistent with top quark pairs has been made using $280 \mathrm{nb}^{-1}$ at $\sqrt{S}_{\mathrm{s}}=7 \mathrm{TeV}$ [10]. 9 candidate events were observed; 7 lepton-jet candidates and 2 di-lepton plus jet candidates. Figure 6 shows the event display for an $e \mu$ candidate event, the isolated electron track is shown as a green track pointing to a green cluster and the muon track is shown in red. The kinematic properties of these events are consistent with top quark pair production but a larger data sample and further studies of control regions are required before a conclusive statement on the observation of top quark production in ATLAS can be made.

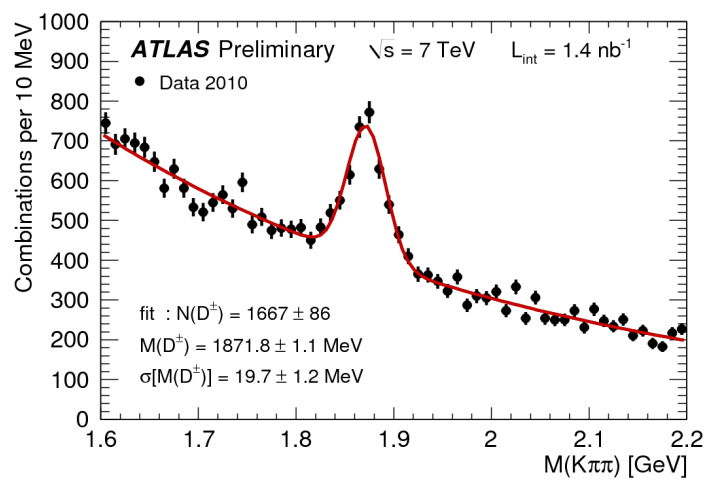

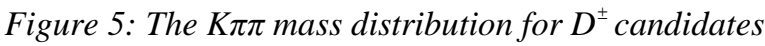
(points).The solid curve represents a fit to the sum of a Gaussian and an exponential background function.

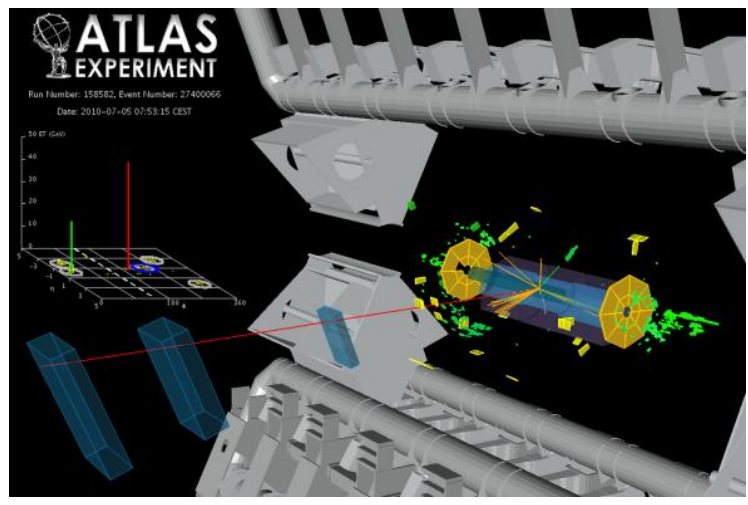

Figure 6.Event display of an ep top quark pair candidate.

\section{References}

[1] ATLAS Collaboration, G. Aad et al., The ATLAS Experiment at the CERN Large Hadron Collider, JINST 3 (2008) S08003

[2] ATLAS Collaboration, Charged-particle multiplicities in pp interactions at $s=900 \mathrm{GeV}$ measured with the ATLAS detector at the LHC, Phys. Lett. B688 (2010) 21-42 [arXiv:1003.312]

[3] ATLAS Collaboration, ATLAS-CONF-2010-024 [http://cdsweb.cern.ch/record/1259231]

[4] ATLAS Collaboration, ATLAS-CONF-2010-031 [http://cdsweb.cern.ch/record/1277665]

[5] ATLAS Collaboration, ATLAS-CONF-2010-046 [http://cdsweb.cern.ch/record/1281338]

[6] ATLAS Collaboration, ATLAS-CONF-2010-050 [http://cdsweb.cern.ch/record/1281302]

[7] ATLAS Collaboration, ATLAS-CONF-2010-049 [http://cdsweb.cern.ch/record/1281299] 
[8] ATLAS Collaboration, ATLAS-CONF-2010-062 [http://cdsweb.cern.ch/record/1281333]

[9] ATLAS Collaboration, ATLAS-CONF-2010-034 [http://cdsweb.cern.ch/record/1277669]

[10] ATLAS Collaboration, ATLAS-CONF-2010-063 [http://cdsweb.cern.ch/record/1281338] 